\title{
Malaria in Pregnancy
}

\author{
Surg Lt Cdr S Chawla*, Surg Lt Cdr V Manu+
}

\begin{abstract}
Background: Malaria in pregnancy contributes to low birth weight and increased infant mortality.

Methods: The study included 416 pregnant women reporting with fever and the impact of malaria on pregnancy was assessed. Result: The study revealed that the protozoal infection affects second trimester more commonly. It increases the chances of abortions, intrapartum foetal distress and meconium stained amniotic fluid.

Conclusion: Malaria is an important cause of feto-maternal morbidity during pregnancy.
\end{abstract}

MJAFI 2007; 63 : 147-148

Key Words: Malaria in pregnancy; Plasmodium

\section{Introduction}

$\mathrm{M}$ alaria remains the most important parasitic disease of man. It kills about 2.5 million people world wide [1]. The impact of malaria during pregnancy includes cerebral malaria, maternal and foetal death due to maternal anaemia and intra uterine growth retardation. Plasmodium is known to have an increased affinity for the decidual vessels of the placenta. Studies have demonstrated that the frequency and severity of malaria is greater in pregnant women with concurrent human immunodeficiency virus (HIV) infection [2]. The identification of the cytoadherence of molecular receptor on the parasitized red blood cell (RBC), has led to the development of vaccine so as to prevent parasite lodging in the placenta [3].

\section{Material and Methods}

A preliminary study was carried out in the service and a civil hospital in the station. The study included 416 pregnant women reporting to the hospital with fever. A detailed history and clinical examination was done to ascertain the cause of fever. Haemoglobin, total and differential leucocyte count, peripheral blood smear for malarial parasite, routine urine examinations were done for all the patients. Additional investigations such as urine culture, sputum for acid-fast bacilli (AFB) etc. were done on the merits of the case. A total of 27 patients were found to be smear positive for plasmodium. The patients were treated with tablet chloroquine. Tablet quinine was to be used once falciparum was detected or in case of complications. Patients who did not respond to quinine were given artesunate. Injectable quinine or artesunate were used in case of vomiting.

\section{Result}

This study included the local population and the dependents of the armed forces personnel. A total of 27 (6.5\%) patients of the 416 women who came with fever were found to be positive for the plasmodium parasite by peripheral blood smear.

Malaria affected all the trimesters of pregnancy, with predominance in the second trimester $(62.9 \%)$, while $22.2 \%$ and $14.9 \%$ were affected in first and third trimesters respectively. Plasmodium falciparum was more common (48.2\%) than the vivax (29.7\%) and other cases had mixed infection. When treated nine patients responded to quinine and two patients required artesunate.

The various complications related to pregnancy in these patients are shown in Table 1. Seven cases were found to have thick meconium stained amniotic fluid (MSAF) when the membranes ruptured spontaneously or artificial rupture of membranes (ARM) was done. These patients underwent

Table 1

Pregnancy complications

\section{Number}

Percentage

Abortion

Ist trimester

IInd trimester

Pre-term labour and low birth weight

$<2.0 \mathrm{~kg}$

$2.0-2.5 \mathrm{~kg}$

Thick MSAF

Intrapartum foetal distress

IUFD (Intra uterine foetal death)

Still birth

7.4
3.7

7.4
7.4
25.9
7.4
3.7
3.7

$2 \quad 7.4$

13.7

27.4

27.4

7
25.9

$2 \quad 7.4$

$1 \quad 3.7$

$1 \quad 3.7$

"Graded Specialist (Obstetrics and Gynaecology) INHS Asvini, Colaba, Mumbai. ${ }^{+}$Graded Specialist (Pathology), INHS Sanjeevani C/o Navy Office, Cochin. 
intrapartum amnioinfusion. Intrapartum foetal distress was observed in two cases. There was one case of intrauterine foetal death (IUFD) at 32 weeks and one case of fresh stillbirth.

Other maternal complications seen during the pregnancy were anaemia in seven patients. One patient presented with cerebral malaria and there were no cases of maternal mortality.

\section{Discussion}

Malaria is a common parasitic infection in humans. Pregnant women are highly susceptible to malaria because of decreased immunity in pregnancy. Malarial parasite lodges itself in the placenta and causes synctial necrosis. It causes thrombosis of the placental vessels leading to infarction. The spikes of fever lead to release of prostaglandins, which causes abortions and preterm labour. The focal placental infarction decreases the nutrition to the foetus causing intrauterine growth retardation, low birth weight and intrauterine foetal death [4,5]. The decreased placental reserves also result in meconium stained amniotic fluid, intrapartum foetal distress and neonatal complications.

The complications of malaria in pregnancy are more common and severe during the first pregnancy. In subsequent pregnancies antibodies prevent cytoadhesion of the plasmodium infected RBC's to the placenta [4].

Maitra et al [6], in their study found plasmodium falciparum affecting $97.2 \%$ of cases and $71 \%$ women were primigravidae. The abortion rate was $100 \%$ in the first trimester and $75 \%$ in the second trimester. The overall foetal loss was $31 \%$. The incidence of maternal death was $8.4 \%$. In this study, there was no maternal death and abortions were seen in $7.4 \%$ and $3.7 \%$ in first and second trimesters respectively. Meconium stained amniotic fluid was seen in $25.9 \%$ of cases. The overall foetal loss in this study was $18.5 \%$. The probable reasons for low foetal loss are regular antenatal visits, close monitoring and availability of better drugs.
Malaria is an important cause for the pregnancy related complications. An early diagnosis and prompt therapy can reduce foetal loss. We feel that all pregnant women coming to the hospital with fever should be screened at the earliest for malarial parasite, especially in the peripheral medical examination room (MI Room)/ primary health centre.

\section{Conflicts of Interest}

None identified

\section{References}

1. Sitalakshmi S, Srikrishna A, Devi S, et al. Changing trends in malaria: A decade's experience at a referral hospital. Indian J Pathol Microbiol 2003; 46: 399-401.

2. Singh N, Saxena A, Shrivastava R. Placental Plasmodium vivax infection and congenital malaria in central India. Ann Trop Med Parasitol 2003; 97: 875-8.

3. McCarthy M. Preventing pregnancy malaria. Simple vaccines that stop parasites lodging in the placenta may save thousands of lives. Lancet 2004; 363: 132-3.

4. Kaushik A, Sharma VK, Sadhana, Kumar R. Malarial placental infection and low birth weight babies. J Common Dis 1992; 24 : 65-9.

5. Singh N, Mehra RK, Srivastava N. Malaria during pregnancy and infancy, in an area of intense malaria transmission in central India. Ann Trop Med Parasitol 2001; 95: 19-29.

6. Maitra N, Joshi M, Hazra M. Maternal manifestations of malaria in pregnancy : A review. Indian J Matern Child Health 1993; 4: 98-101.

7. Martinez-Espinosa FE, Daniel-Ribeiro CT, Alecrim WD. Malaria during pregnancy in a reference centre from the Brazilian Amazon: unexpected increase in the frequency of Plasmodium falciparum infections. Mem Inst Oswasldo Cruz 2004; 99: 19-21.

8. McGready R, Cho T, Cho JJ, et al. Artemisinin derivatives in the treatment of falciparum malaria in pregnancy. Trans R Soc Trop Med Hyg 1998; 92: 430-3.

9. Deen JL, Von Seidlein L, Pinder M, et al. Safety of combination artesunate and pyrimethamine-sulfadoxine given during pregnancy. Trans R Soc Trop Med Hyg 2001; 95: 424-8.

\section{ANNOUNCEMENT BEST REFEREE AWARD : MJAFI}

The Best Referee Award has been instituted with effect from 2006 to appreciate the contribution made by the referees in publication of the articles in MJAFI. The award will be given during the AFMRC annually. The criteria for selection of Best Referee would be:
(a) Usage of email in all correspondence
(b) First response within two weeks of receipt of article
(c) Subsequent responses within one week
(d) Most constructive comments 